\title{
Research Article \\ Optimizing Training Set Construction for Video Semantic Classification
}

\author{
Jinhui Tang, ${ }^{1}$ Xian-Sheng Hua, ${ }^{2}$ Yan Song, ${ }^{1}$ Tao Mei, $^{2}$ and Xiuqing Wu ${ }^{1}$ \\ ${ }^{1}$ Department of Electronic Engineering and Information Science, University of Science and Technology of China, Hefei 230027, China \\ ${ }^{2}$ Microsoft Research Asia, Beijing 100080, China
}

Correspondence should be addressed to Jinhui Tang, jhtang@mail.ustc.edu.cn

Received 9 March 2007; Revised 14 September 2007; Accepted 12 November 2007

Recommended by Mark Kahrs

We exploit the criteria to optimize training set construction for the large-scale video semantic classification. Due to the large gap between low-level features and higher-level semantics, as well as the high diversity of video data, it is difficult to represent the prototypes of semantic concepts by a training set of limited size. In video semantic classification, most of the learning-based approaches require a large training set to achieve good generalization capacity, in which large amounts of labor-intensive manual labeling are ineluctable. However, it is observed that the generalization capacity of a classifier highly depends on the geometrical distribution of the training data rather than the size. We argue that a training set which includes most temporal and spatial distribution information of the whole data will achieve a good performance even if the size of training set is limited. In order to capture the geometrical distribution characteristics of a given video collection, we propose four metrics for constructing/selecting an optimal training set, including salience, temporal dispersiveness, spatial dispersiveness, and diversity. Furthermore, based on these metrics, we propose a set of optimization rules to capture the most distribution information of the whole data using a training set with a given size. Experimental results demonstrate these rules are effective for training set construction in video semantic classification, and significantly outperform random training set selection.

Copyright (C) 2008 Jinhui Tang et al. This is an open access article distributed under the Creative Commons Attribution License, which permits unrestricted use, distribution, and reproduction in any medium, provided the original work is properly cited.

\section{INTRODUCTION}

Video content analysis is an elementary step for mining the semantic information in video collections, in which semantic classification (or we may call it annotation) of video segments is essential for further analysis, as well as important for enabling semantic-level video search. For human being, most semantic concepts are clear and easy to identify, while due to the large gap between semantics and low-level features, the corresponding features generally are not well-separated in feature space thus difficult to be identified by computer. This is an open difficulty in computer vision and visual content analysis area.

Generally, learning-based video semantic classification methods use statistical learning algorithms to model the semantic concepts (generative learning) or the discriminations among different concepts (discriminative learning). In [1], hidden Markov model and dynamic programming are applied to play/break segmentation in soccer videos. Fan et al. [2] classify semantic concepts for surgery education videos by using Bayesian classifiers with an adaptive EM algorithm. Zhong and Chang [3] propose a unified framework for scene detection and structure analysis by combining domainspecific knowledge with supervised machine learning methods. However, most of these learning-based approaches require a large training set to achieve good generalization capacity, thus a great deal of labor-intensive manual labeling is inevitable. On the other hand, semisupervised learning techniques, which try to exploit the information embedded in unlabeled data, are proposed to improve the performance. In [4], cotraining is applied to video annotation based on a careful split of visual features. Yan and Naphade [5] point out the drawbacks of cotraining in video annotation, and propose an improved cotraining style algorithm named semisupervised cross-feature learning. An structure-sensitive manifold ranking method is proposed in [6] for video concept detection, where the authors analyze the graph-based semisupervised learning methods from the view of PDEbased diffusion. Tang et al. [7] embed the temporal consistency of video data into the graph-based SSL and propose 
a temporally consistent Gaussian random field method for video annotation. A method based on kernel density estimation is proposed in [8] for video semantic detection, where the authors show that this method has close relationship with the graph based semisupervised learning. In addition, active learning scheme is also an effective solution to this problem $[9,10]$. However, all these methods have paid little attention on the issue of the training set construction. Generally, most of them adopt a random selection scheme to construct the training set. In this paper, we argue that a better training set, though the size is very small, can be carefully constructed/selected with a good performance being simultaneously preserved.

It has been shown that the generalization capacity of a classifier usually depends on the geometrical distribution of the training data rather than the size [11]. Therefore, if the selected training data can capture this kind of characteristic of the entire video collection, the classification performance will still be good enough even in the case that the size of training set is much smaller than that of the whole dataset, thus much manual labor for training data labeling will be saved. In other words, according to the distribution analysis of the video dataset, a "skeleton" of the prototypes of the semantic concepts can be achieved in a training set with an extremely limited number of samples.

Given a large video collection, it is possible to construct a small-size but effective training set (to be labeled manually) by exploiting the temporal and spatial distribution of the entire dataset. Typically, a semantic concept and its corresponding feature variations within the same video are relatively smaller than those among different videos and the concept drifting is gradual in most cases [12]. The clustering information can be extracted according to this observation. That is, based on visual similarity and temporal order, the video shots can be preclustered in an over-segmentation manner [4]. Each cluster can be represented by the cluster center (or the shot closest to the cluster center in terms of low-level features). This clustering process aims at making all the samples within each cluster most likely associate with the same semantic concept. As a result, the training set can be constructed by selecting samples from these cluster centers. Intuitively, we can take all the cluster centers as the training set. However, as clustering information is obtained in an over-segmentation manner, typically the number of cluster centers is very large. Therefore, much redundancy still exists among these clusters and actually only a small part of them is highly informative.

In this paper, we analyze the factors which can capture the distribution characteristics of a given video collection, and propose the following four metrics for the training set construction, including salience, temporal dispersiveness, spatial dispersiveness, and diversity. First, as the candidates for constructing the training set are actually cluster centers, the samples in this candidate set should have different potential contributions to the training set as their corresponding cluster sizes are different. Accordingly, we introduce salience, as a potential contribution measure of each candidate sample. Second, the samples in the training set should distribute dispersively in temporal order, as well as in the low-level feature

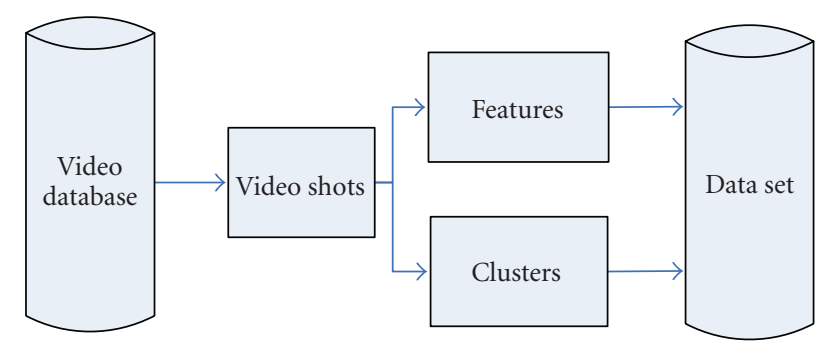

Figure 1: Preprocessing of video database.

space, thus more "prototypes" of the semantic concept can be selected. Therefore, we introduce two measures, temporal dispersiveness and spatial dispersiveness, to reflect how well the training set captures the distribution of the entire video dataset in temporal order and the feature space, respectively. Finally, in addition to temporal and spatial dispersiveness, the selected samples need to be diversely distributed in the feature space [13]. In this paper, the measure diversity is defined to capture this training set property.

According to the above analyses, a set of optimization rules based on these metrics are further proposed to reduce the redundancy in the set of cluster centers. A set of experiments are conducted on a real-video dataset to show the effectiveness of these rules.

The rest of this paper is organized as follows. In Section 2, representativeness metrics for training set construction are presented. Section 3 discusses the optimization rules and methods according to the representativeness metrics. Experimental results are presented in Section 4, followed by concluding remarks and future work in Section 5.

\section{REPRESENTATIVENESS METRICS}

In this section, we first describe the preprocessing step of video database, including shot detection, feature extraction, and preclustering. Then the four metrics including salience, temporal dispersiveness, spatial dispersiveness, and diversity are discussed in detail based on the preprocessing results.

Figure 1 illustrates the flowchart of preprocessing the video dataset. First, each video is segmented into shots according to timestamp (for DVs) or visual similarity (for ana$\log$ videos). In the following process, each shot is represented by a certain number of frames uniformly excerpted from the shot. Shot is taken as the elementary unit for the semantic classification in this paper, which is the basic annotation unit most frequently applied in the literature.

All the shots in the video database are preclustered based on their visual similarity measure and temporal order in an over-segmentation manner, in which all the shots belonging to a certain cluster mostly correspond to the same semantic concept [4]. Then, in the process of classification, one cluster is taken as one sample, instead of using one shot as an individual sample, which can significantly reduce the number of shots that need to be labeled by users [14]. Yuan et al. [15] also show that simply taking cluster centers for training works well with theoretical insight. Here our objective 


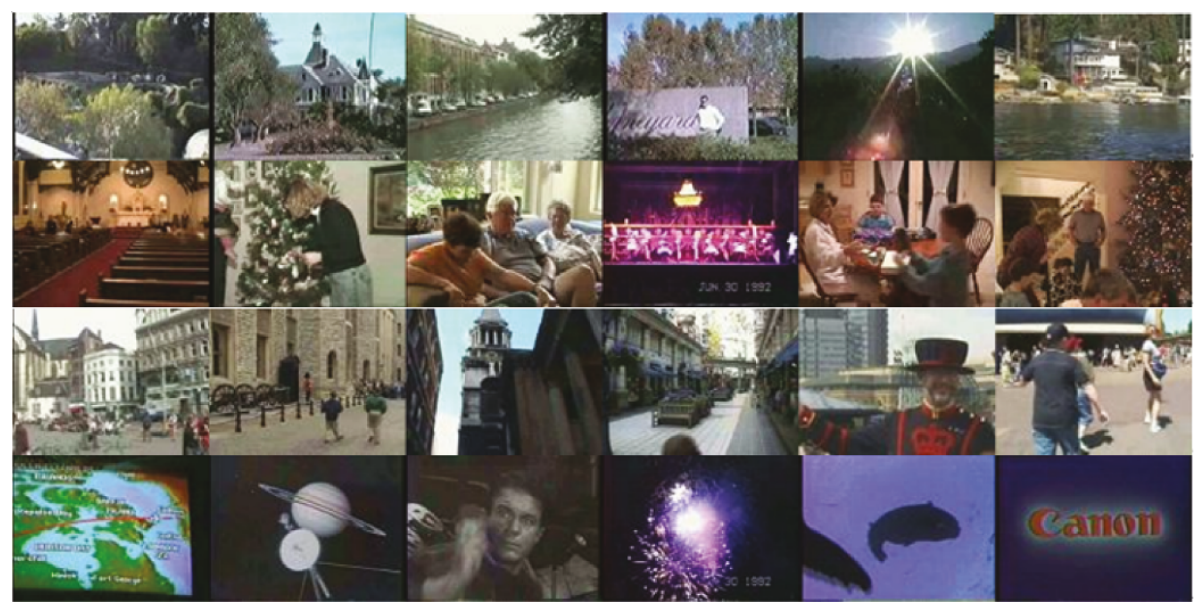

FIGURE 2: Exemplary thumbnails for the 4 different sematic classes. First row: landscape; second row: indoor; third row: cityscape; last row: others.

is different from theirs. We aim to select a set of informative samples for the users to annotate and then the set is used for training. Before the training set being constructed, the labels are unknown, and they use the labels of the entire dataset. Our objective is to reduce the manual work while Yuan's work focuses on reducing the number of support vectors.

As aforementioned, the training set is constructed to roughly represent the prototypes of the semantic concepts to be modeled from the video collection. Here, we detail the aforementioned four metrics to measure the representativeness of a training set. To clearly present our ideas, we define the following notations at first.

Notation 1. The center (or representative shot) set of the clusters is denoted by CntSet $=\left\{x_{j}, 1 \leq j \leq K(\mathrm{cl})\right\}$, where $x_{j}$ is the shot closest to the center of the $j$ th cluster and $K(\mathrm{cl})$ is the total number of the clusters in the whole video dataset.

Notation 2. The training set consisted of the selected shots from CntSet is denoted by TrnSet $=\left\{x_{i}, 1 \leq i \leq M\right\}$, where $M$ is the size of training set that will be constructed. TrnSet is a subset of CntSet.

Notation 3. The distance between two sample feature vectors in the kernel mapped feature space is defined as $\operatorname{dis}\left(\phi_{i}, \phi_{j}\right)$ :

$$
\begin{aligned}
\operatorname{dis}\left(\phi_{i}, \phi_{j}\right) & =\left\|\phi\left(x_{i}\right)-\phi\left(x_{j}\right)\right\|=\sqrt{\phi_{i}^{T} \phi_{i}-2 \phi_{i}^{T} \phi_{j}+\phi_{j}^{T} \phi_{j}} \\
& =\sqrt{K\left(x_{i}, x_{i}\right)-2 K\left(x_{i}, x_{j}\right)+K\left(x_{j}, x_{j}\right)},
\end{aligned}
$$

where $\phi_{i}$ is the kernel mapping of the feature vector $x_{i}$ (we use $x$ to denote both the shot and its feature vector in this paper), $K$ is the kernel function. In our experiments, Gaussian kernel is adopted for $K$.

Based on these notations, we introduce four metrics to measure the effectiveness of a training set.

\subsection{Salience metric}

First, the effectiveness of samples (cluster centers) is different from each other, that is, the sample corresponding to a large cluster should be more "important" than the ones of small clusters. In other words, such samples most likely represent the salient prototypes of the semantic concepts. Therefore, we define SAL as the salience metric of TrnSet as follows.

Metric 1. Salience:

$$
\mathrm{SAL}=\frac{1}{K(\mathrm{cl})} \sum_{x_{i} \in \operatorname{TrnSet}} \operatorname{Sal}\left(x_{i}\right)
$$

where $\operatorname{Sal}\left(x_{i}\right)$ is the number of shots in the cluster corresponding to the $i$ th sample in TrnSet.

\subsection{Temporal dispersiveness metric}

Second, the samples to be selected should distribute dispersively through the temporal axis of the whole video dataset. Thus more prototypes of the semantic concept can be preserved. This is from the observation that if the two salient samples lie close to each other in temporal order, it may belong to the same concept with high probability. We define the temporal distance between the sets CntSet and TrnSet as

$$
\operatorname{Dis}_{T}=\frac{1}{K(\mathrm{cl})} \sum_{x_{j} \in \mathrm{CntSet}} \min _{x_{i} \in \operatorname{TrnSet}}\left\|t\left(x_{i}\right)-t\left(x_{j}\right)\right\|,
$$

where $\min _{x_{i} \in \operatorname{TrnSet}}\left\|t\left(x_{i}\right)-t\left(x_{j}\right)\right\|$ is the temporal distance between $x_{j}$ and TrnSet, and $t(x)$ is the normalized temporal order of the sample $x$. The temporal dispersiveness is defined as follows.

\section{Metric 2. Temporal dispersiveness:}

$$
T \_ \text {Disp }=\frac{1}{\operatorname{Dis}_{T}}=\frac{K(\mathrm{cl})}{\sum_{x_{j} \in \mathrm{CntSet}} \min _{x_{i} \in \operatorname{TrnSet}}\left\|t\left(x_{i}\right)-t\left(x_{j}\right)\right\|} .
$$




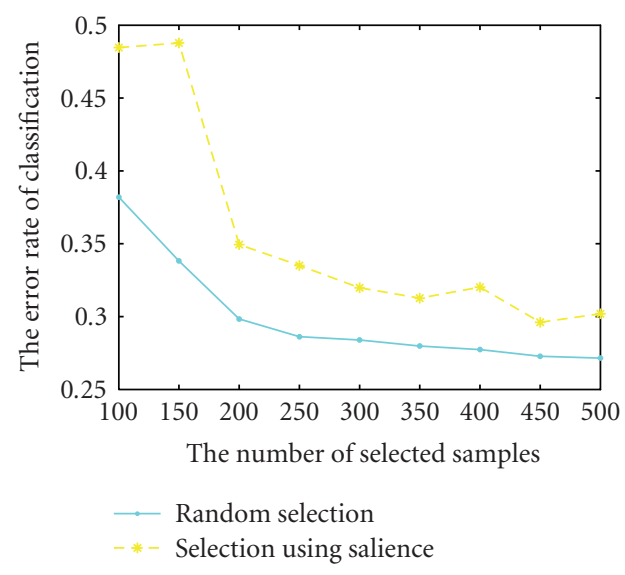

(a)

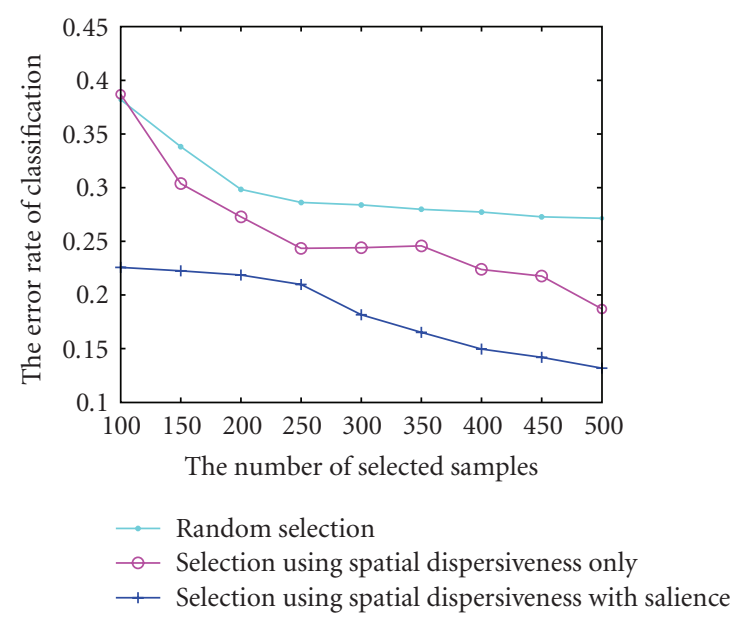

(c)

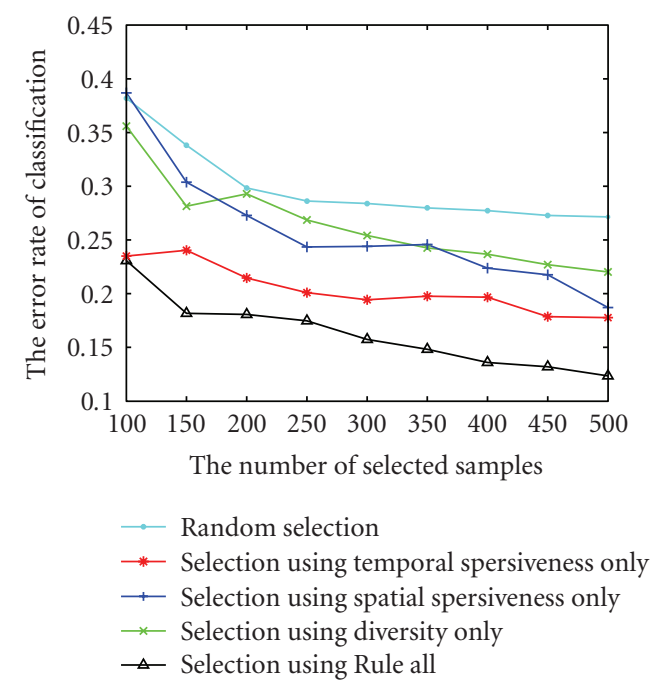

(e)

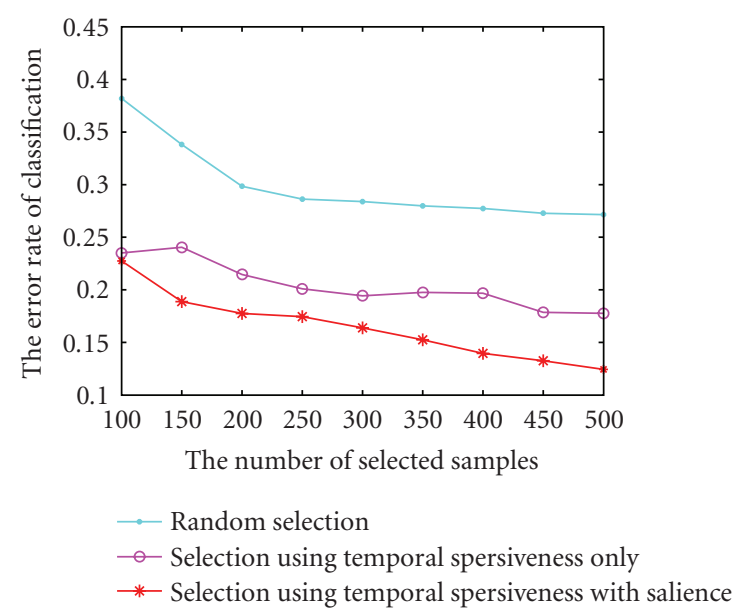

(b)

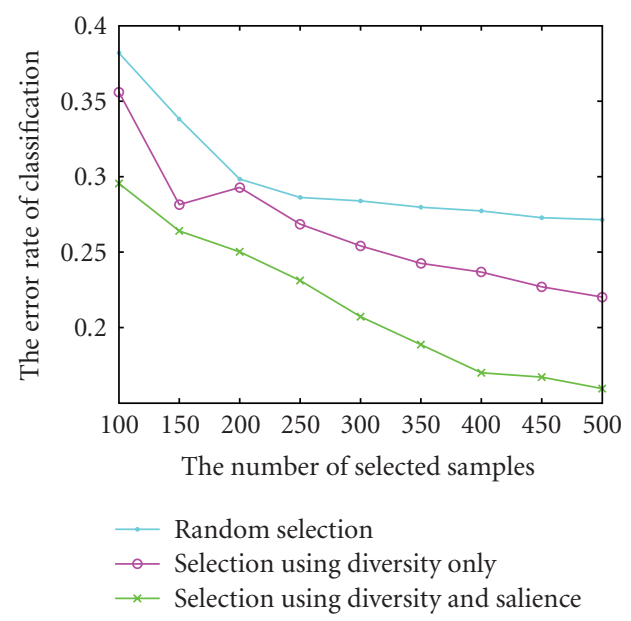

(d)

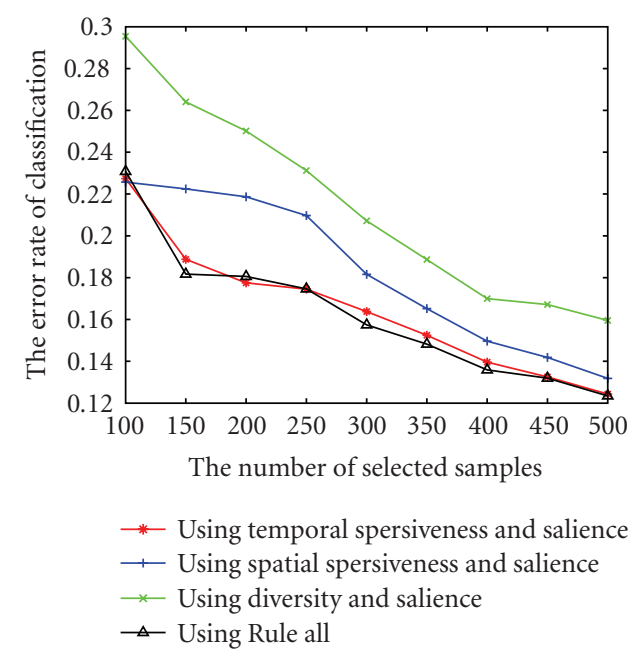

(f)

FIgURE 3: Comparisons of the experimental results in a transductive manner. 


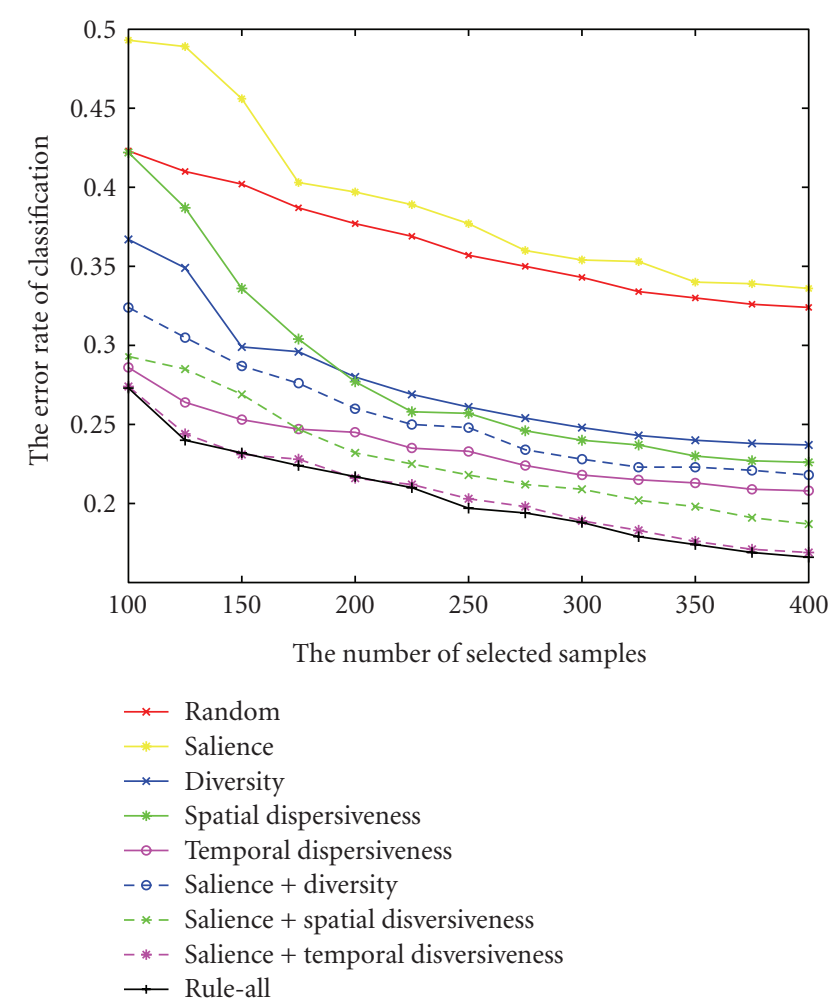

FIGURE 4: Comparisons of the experimental results after data separation.

In order to assure that the TrnSet can capture most temporal distribution information of the CntSet, it is necessary to minimize the $\mathrm{Dis}_{T}$, which is equivalent to the maximiza-

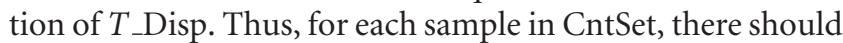
be a sample in TrnSet close to it in temporal order. Given the size of the TrnSet, maximizing T_Disp can mostly disperse the samples in TrnSet in temporal order.

\subsection{Spatial dispersiveness metric}

Third, similar to the aforementioned temporal dispersiveness, the samples to be selected should distribute dispersively through the whole kernel mapped feature space. This is from the observation that if the two salient samples lie close to each other in the feature space, it may belong to the same concept with high probability. We define the spatial distance Dis between the sets CntSet and TrnSet as

$$
\operatorname{Dis}_{S}=\frac{1}{K(\mathrm{cl})} \sum_{x_{j} \in \mathrm{CntSet}} \min _{x_{i} \in \operatorname{TrnSet}}\left\|\phi\left(x_{i}\right)-\phi\left(x_{j}\right)\right\|,
$$

where $\min _{x_{i} \in \operatorname{TrnSet}}\left\|\phi\left(x_{i}\right)-\phi\left(x_{j}\right)\right\|$ is the spatial distance between $x_{j}$ and TrnSet. Then we define spatial dispersiveness as follows.

Metric 3. Spatial dispersiveness:

$$
S_{\_} \text {Disp }=\frac{1}{\operatorname{Dis}_{S}}=\frac{K(\mathrm{cl})}{\sum_{x_{j} \in \mathrm{CntSet}} \min _{x_{i} \in \operatorname{TrnSet}}\left\|\phi\left(x_{i}\right)-\phi\left(x_{j}\right)\right\|},
$$

where $\phi(x)$ is the kernel mapping of $x$. TrnSet can capture the most spatial distribution characteristics of CntSet through maximizing $S \_D i s p$. It corresponds to minimizing Dis $S_{S}$, that is, the samples in CntSet have a minimal average distance to TrnSet in the kernel mapped space. Thus, for each sample $x_{j}$ in CntSet, there should be a sample in TrnSet close to it. Given the size of TrnSet, maximization of $S_{-}$Disp can mostly disperse the samples in TrnSet in the mapped feature space.

\subsection{Diversity metric}

Goh et al. [13] have pointed out that the selected samples need to be diversified in image retrieval application, and defined the measure angle diversity to choose the sample with the maximal angle (less than $90^{\circ}$ ) to the current selected sample set. That is, the selected sample should be "almost orthogonal" to current selected sample set. However, their definition of the angle between the unlabeled instance $x_{i}$ to the current sample set $S$ is the maximal angle from instance $x_{i}$ to any instance $x_{j}$ in set $S$. This definition just ensures that the chosen instances can be almost orthogonal to one sample in current set, but not almost orthogonal to the set. We introduce feature vector selection (FVS) method to handle this problem. FVS method is proposed in [16] to find an approximate basis of the whole dataset to reduce feature dimension. Here we employ it to find the almost orthogonal sample set in CntSet. FVS is similar to the kernel principal component analysis (KPCA) while FVS selects the existed sample vectors as the basis, and the KPCA uses the first $k$ eigenvectors as the basis. The authors of [16] show that in some special cases the FVS-PCA is equivalent to KPCA.

As aforementioned, the samples in TrnSet are denoted as $\left\{x_{i}, 1 \leq i \leq M\right\}$, where $M$ is the size of TrnSet. Given a wellselected TrnSet, each sample $x_{j}$ in CntSet could be approximated by the linear combination of samples in TrnSet in the kernel mapped space. The normalized Euclidean distance $\delta_{j}$ is defined to measure the fitness between $\phi\left(x_{j}\right)$ and $\hat{\phi}\left(x_{j}\right)$ as follows:

$$
\delta_{j}=\frac{\left\|\phi\left(x_{j}\right)-\hat{\phi}\left(x_{j}\right)\right\|^{2}}{\left\|\phi\left(x_{j}\right)\right\|^{2}}
$$

$\delta_{j}$ is a similarity measure between the original vector $\phi\left(x_{j}\right)$ and the reconstructed vector $\hat{\phi}\left(x_{j}\right)=\sum_{x_{i} \in \operatorname{TrnSet}} \alpha_{j i} \phi\left(x_{i}\right)$. The smaller $\delta_{j}$ is, the better $x_{j}$ can be approximated by TrnSet. Consequently, the metric diversity can be defined as follows.

Metric 4. Diversity:

$$
\begin{aligned}
\text { Divers } & =1-\frac{1}{K(\mathrm{cl})} \sum_{x_{j} \in \mathrm{Cn} t S e t} \delta_{j} \\
& =1-\frac{1}{K(\mathrm{cl})} \sum_{x_{j} \in \mathrm{Cn} t S e t} \frac{\left\|\phi\left(x_{j}\right)-\sum_{x_{i} \in \operatorname{TrnSet}} \alpha_{j i} \phi\left(x_{i}\right)\right\|^{2}}{\left\|\phi\left(x_{j}\right)\right\|^{2}}
\end{aligned}
$$

where $a_{i j}$ are weights of the combination. This metric demonstrates how the TrnSet can capture the diversity of CntSet. Given the size of TrnSet, maximization of Divers can 
lead the samples in TrnSet to be almost orthogonal to each other. It is worth noting that the aim of spatial dispersiveness is to distribute the selected samples in the feature space with maximal average distance under L1 norm. It is similar to minimize the reconstruction error with the only closest sample under L1 norm. The aim of diversity is to minimize the linear reconstruction error under the L2 norm. They are similar but have difference.

\section{OPTIMIZATION RULES}

As aforementioned, four metrics have been defined to measure the representativeness of TrnSet. According to these metrics, the following rules are further proposed to construct an optimal training set with a given size.

Rule 1. Maximizing salience:

$$
\text { TrnSet }^{*}=\underset{\text { TrnSet } \subset \text { CntSet }}{\operatorname{argmax}}\{\mathrm{SAL} \mid \#(\text { TrnSet })=\mathrm{M}\}
$$

where \#(TrnSet) is the number of samples in $\operatorname{TrnSet}, M$ is a given number.

The constructing procedure based on this rule can be described as shown in Algorithm 1.

Rule 2. Maximizing temporal dispersiveness:

$$
\text { TrnSet }^{*}=\underset{\text { TrnSetCCntSet }}{\arg \max }\left\{T \_ \text {Disp } \mid \#(\text { TrnSet })=M\right\} .
$$

This rule is equal to minimize $\mathrm{Dis}_{T}$, and the training set construction procedure is illustrated in Algorithm 2.

Rule 3. Maximizing spatial dispersiveness:

$$
\text { TrnSet }^{*}=\underset{\text { TrnSetcCntSet }}{\arg \max }\left\{S_{-} \text {Disp } \mid \#(\text { TrnSet })=M\right\}
$$

This rule is equal to minimize $\mathrm{Dis}_{S}$, and the procedure can be accomplished similar to Rule 2 , just needs to change the temporal distance $d t_{m n}$ to the spatial distance dis $\left(\phi_{m}, \phi_{n}\right)=$ $\left\|\phi\left(x_{m}\right)-\phi\left(x_{n}\right)\right\|$.

Rule 4. Maximization of diversity:

$$
\text { TrnSet }^{*}=\underset{\text { TrnSet } \subset \text { CntSet }}{\operatorname{argmax}}\{\text { Divers } \mid \#(\text { TrnSet })=\mathrm{M}\}
$$

So the target is to find a set (TrnSet) of feature vectors (FVs) [16] with the fixed size which minimize

$$
\sum_{x_{j} \in \text { CntSet }} \frac{\left\|\phi\left(x_{j}\right)-\sum_{x_{i} \in \operatorname{TrnSet}} \alpha_{j i} \phi\left(x_{i}\right)\right\|^{2}}{\left\|\phi\left(x_{j}\right)\right\|^{2}} .
$$

It has been proven in [16] that the minimization of

$$
\delta_{j}=\frac{\left\|\phi\left(x_{j}\right)-\hat{\phi}\left(x_{j}\right)\right\|^{2}}{\left\|\phi\left(x_{j}\right)\right\|^{2}}
$$

for a given size $M$ of FVs can be expressed with dot products only:

$$
\min \delta_{j}=1-\frac{K_{S j}^{T} K_{S S}^{-1} K_{S j}}{k_{j j}},
$$

where

$$
K_{S S}=\left[K\left(x_{p}, x_{q}\right)\right]_{1 \leq p, q \leq M}
$$

is a square matrix of dot products of FVs, and

$$
K_{S j}=\left[K\left(x_{p}, x_{j}\right)\right]_{1 \leq p \leq M}
$$

is the vector of dot products between $x_{j}$ and the FVs.

Define the fitness for the sample $x_{j}$ by

$$
J_{S_{j}}=\frac{\left\|\hat{\phi}\left(x_{j}\right)\right\|^{2}}{\left\|\phi\left(x_{j}\right)\right\|^{2}}=\frac{K_{S j}^{T} K_{S S}^{-1} K_{S j}}{k_{j j}},
$$

which is a measure of the best fit case, where $x_{j} \in \mathrm{CntSet}$, $x_{i} \in \operatorname{TrnSet}$ and $k_{j j}=K\left(x_{j}, x_{j}\right)$. Then the objective becomes to select a set TrnSet for a given size $M$ to maximize the fitness for the CntSet:

$$
J S=\frac{1}{K(\mathrm{cl})} \sum_{x_{j} \in \mathrm{CntSet}} J_{S_{j}}
$$

Note that the maximum of (19) is one and for $x_{i} \in$ TrnSet, (15) is zero. Therefore, when \#(TrnSet) increases, we only need to explore $(K(l)-\#(\operatorname{TrnSet}))$ remaining vectors to evaluate the maximization of $J S$.

The process is iterative, which consists of a set of sequential forward selection operations: at the first iteration, we look for the sample that gives the maximum JS. Except for the first iteration, the algorithm uses the lowest fitness $J_{S_{j}}$ for the current basis TrnSet to select the new FV while evaluating the JS. JS is monotonic since the new basis will reconstruct all the samples at least as well as the previous basis did. Algorithm 3 shows the detailed procedure.

Among the four metrics, salience is the property of each sample, while the other three metrics are related to the correlations between TrnSet and CntSet. Therefore, salience can be combined into Rule 2-4 to improve the results.

Rule $1+2$. Maximizing temporal dispersiveness with salience. ${ }^{1}$ We want the sample with high salience to have more chance to be selected, so we can minimize

$$
\sum_{x_{j} \in \mathrm{CntSet}} \min _{x_{i} \in \operatorname{TrnSet}} \frac{\left\|t\left(x_{i}\right)-t\left(x_{j}\right)\right\|}{\operatorname{Sal}\left(x_{i}\right) \cdot \operatorname{Sal}\left(x_{j}\right)}
$$

subject to a fixed-size TrnSet. The training set construction procedure of this rule is presented in Algorithm 4.

\footnotetext{
${ }^{1}$ The computation for optimizing Rule $1+2$ is NP hard. For approximation, we remove the samples, which are not dispersive and salient either, from the CntSet. Thus, the distance measure defined in step 2 of Algorithm 4 is different from the definition in (20). The optimizations of Rule $1+3$ and Rule 1-3 also have this case.
} 
1: Initialization: TrnSet $:=\{\}$ and $\#($ TrnSet $)=0$;

2: Obtain $\operatorname{Sal}\left(x_{j}\right)$ for every sample $x_{j}$ in CntSet according to the cluster size;

3: While \# (TrnSet $)<M$

Find the maximal element maxSal in vector $\left[\operatorname{Sal}\left(x_{j}\right)\right]_{1 \leq j \leq K(\mathrm{cl})}$;

Add the sample corresponding to maxSal to TrnSet;

Remove this sample from CntSet;

$\#($ TrnSet $)=\#($ TrnSet $)+1$;

End While

4: Return TrnSet

Algorithm 1: Optimization of Rule 1.

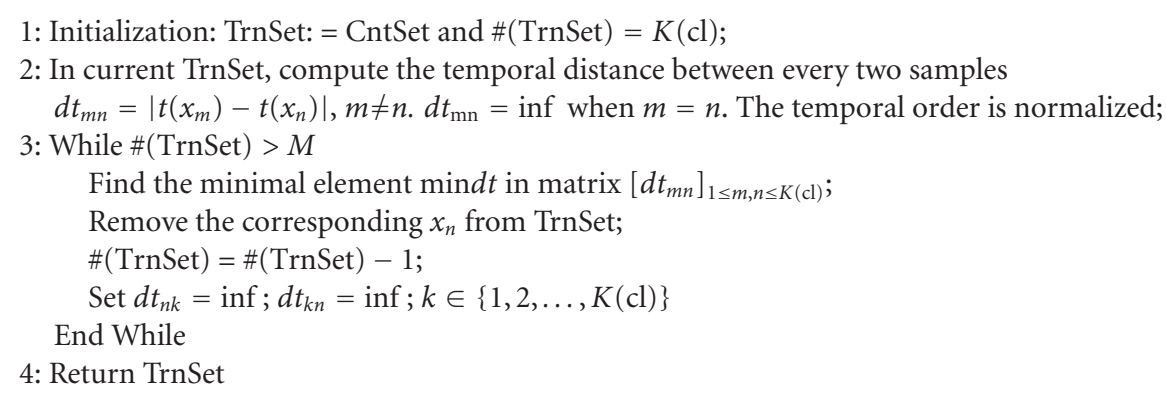

Algorithm 2: Optimization of Rule 2.

Rule $1+3$. Maximizing spatial dispersiveness with salience. Similar to Rule $1+2$, we minimize

$$
\sum_{x_{j} \in \mathrm{CntSet}} \min _{x_{i} \in \operatorname{TrnSet}} \frac{\left\|\phi\left(x_{i}\right)-\phi\left(x_{j}\right)\right\|}{\operatorname{Sal}\left(x_{i}\right) \cdot \operatorname{Sal}\left(x_{j}\right)}
$$

subject to a fixed-size TrnSet. This procedure is similar to Rule $1+2$.

Rule $1+4$. Maximizing diversity accompanied with salience.

Consider the effect of salience, the objective becomes finding a feature vector set (FVs) under the constraint of fixed size to minimize

$$
\sum_{x_{j} \in \operatorname{CntSet}} \frac{\left\|\phi\left(x_{j}\right)-\sum_{x_{i} \in \operatorname{TrnSet}} \alpha_{j i} \phi\left(x_{i}\right)\right\|^{2}}{\left\|\phi\left(x_{j}\right)\right\|^{2} \cdot \operatorname{Sal}\left(x_{j}\right)} .
$$

Then we can select samples as the procedure in Algorithm 5.

Actually, finally, we want to use all these four metrics to optimize TrnSet. A direct way is to maximize a linear combination of the four metrics, that is, to maximize

$$
\begin{aligned}
R= & \alpha \cdot \mathrm{SAL}+\beta \cdot T \_ \text {Disp } \\
& +\gamma \cdot \mathrm{S} \_ \text {Disp }+(1-\alpha-\beta-\gamma) \cdot \text { Divers }
\end{aligned}
$$

subject to a fixed-size TrnSet. However, it is not easy to determine the three weights (which is our future work). Alterna- tively, in this paper, we optimize the four metrics in a hierarchical way. That is, firstly we minimize

$$
\sum_{x_{j} \in \mathrm{CntSet}} \min _{x_{i} \in \operatorname{TrnSet}} \frac{\operatorname{dis}\left(\phi_{i}, \phi_{j}\right) \cdot d t\left(x_{i}, x_{j}\right)}{\operatorname{Sal}\left(x_{i}\right) \cdot \operatorname{Sal}\left(x_{j}\right)}
$$

to optimize the Metric 1-3 simultaneously (see Algorithm 6), and then use Rule $1+4$ to remove $10 \%$ redundancy. We call this method Rule_all.

\section{EXPERIMENTAL RESULTS}

To evaluate the performance of our proposed algorithms on real video dataset, we conduct several experiments on a home video dataset which contains about 55 home videos with a wide variety of contents, such as wedding, vacation, meeting, party, and sports.

In the experiments, we classify the shots in the video dataset into the following four semantic concepts: indoor, landscape, cityscape, and others. The four semantic concepts are mutually exclusive, that is, one sample just can belong to one concept. After preprocessing of the video dataset including shot detection, low-level feature extraction and preclustering, about 7000 shots are obtained. These shots are further clustered into about 1600 clusters in an over-segmentation manner. Each shot is labeled as indoor, cityscape, landscape, and others according to the definitions in TRECVID [17]. Some exemplary thumbnails of these concepts are shown in Figure 2. 


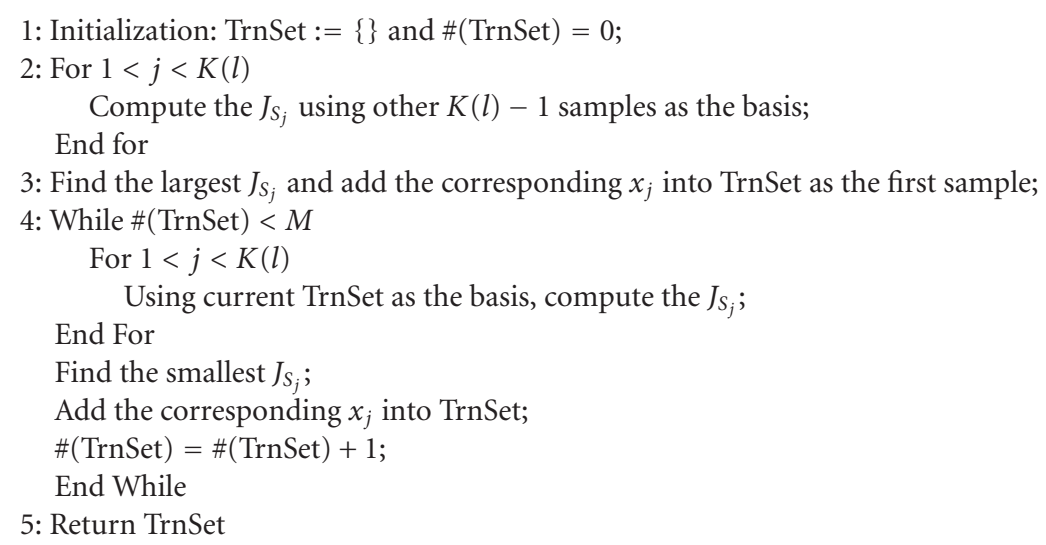

Algorithm 3: Optimization of Rule 4.

1: Initialization: $\operatorname{TrnSet}=\mathrm{CntSet}$ and $\#(\operatorname{TrnSet})=K(\mathrm{cl})$;

2: In current TrnSet, compute the following distance between every two samples

$d t_{m n}=\operatorname{Sal}\left(x_{m}\right) \cdot \operatorname{Sal}\left(x_{n}\right) \cdot d t\left(x_{m}, x_{n}\right), m \neq n . d_{m, n}=\inf$ when $m=n$. The temporal order is normalized; 3: While \# (TrnSet) $>M$

Find the minimal element $\min \_d t$ in matrix $\left[d t_{m n}\right]_{1 \leq m, n \leq K(\mathrm{cl})}$;

Find the corresponding $x_{m}$ and $x_{n}$;

If $\operatorname{Sal}\left(x_{m}\right) \geq \operatorname{Sal}\left(x_{n}\right)$

Remove $x_{n}$ from TrnSet;

Set $d_{n k}=\inf ; d_{k n}=\inf ; k \in\{1,2, \ldots, K(\mathrm{cl})\}$

Else

Remove $x_{m}$ from TrnSet;

Set $d_{m k}=\inf ; d_{k m}=\inf ; k \in\{1,2, \ldots, K(\mathrm{cl})\}$

End If

End While

4: Return TrnSet

Algorithm 4: Optimization of Rule $1+2$.

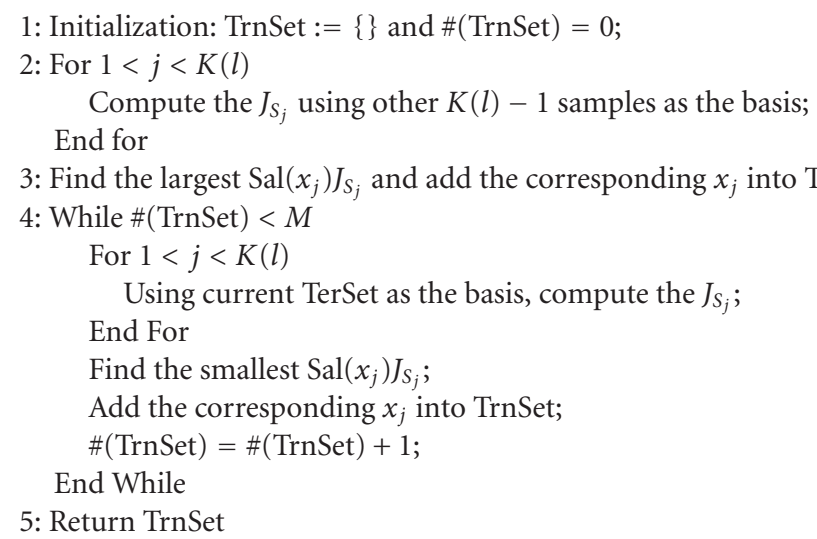




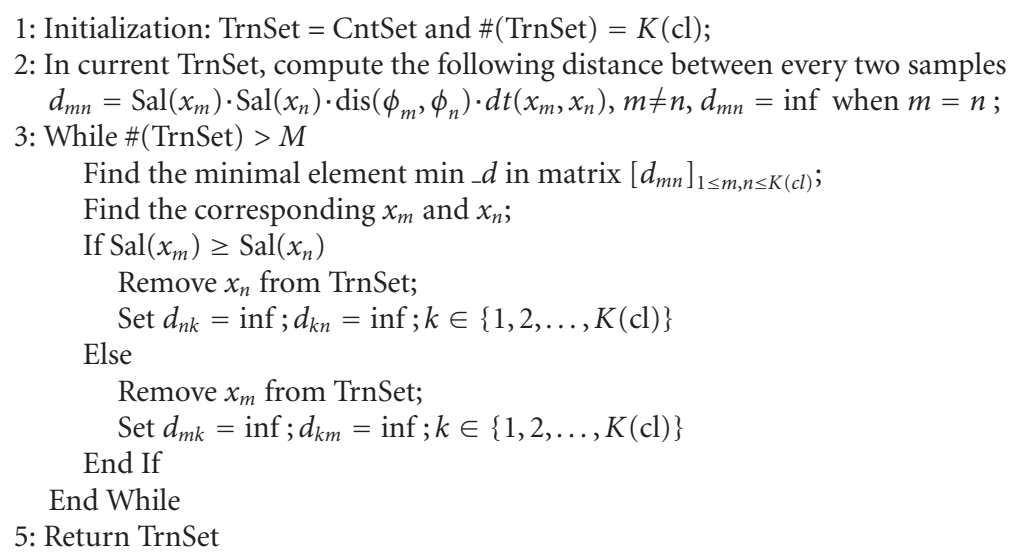

Algorithm 6: Optimization of Rule 1-3.

The low-level features we used here has 90 dimensions, consisting of a 36-D HSV color histogram, a 9D color moment, and a 45D blockwise edge distribution histogram. Low-level features are normalized by Gaussian normalization [18]. Each shot is represented by a certain number (i.e., 10) of frames uniformly excerpted from the shot, and the shot closest to the cluster center is taken as the sample to form the dataset. So the dataset used in experiment has about 7000 samples, and each sample is represented as a 900D vector. The CntSet has about 1600 samples, and each sample is also a $900 \mathrm{D}$ vector.

We conduct 5 experiments in transductive manner: when the training set TrnSet is constructed, we train the SVM model [19] to classify the samples in CntSet (here the parameters $C$ and $g$ are both set to 1 empirically), and then extend the label of each cluster center to all other samples in the same cluster [14]. The error rates are calculated for all samples on all concepts.

Experiment 1. Construct the training set using Rule 1. The classification error rate is illustrated in Figure 3(a), compared with random training set selection (averaged over ten runs). We can see that the result is worse than the random selection. That is because the distribution information of original data is significantly lost in the training set constructed by using Rule 1 only.

Experiment 2. Construct the training set using Rule 1 and Rule $1+2$. The results are shown in Figure 3(b). It can be seen that Rule 2 significantly improves the classification performance and the embedding of salience further improves Rule 2.

Experiment 3. Construct the training set using Rule 3 and Rule $1+3$. The results in Figure 3(c) show that Rule 3 also improves the classification performance significantly. And it is effective to embed salience into Rule 3.

Experiment 4. Construct the training set using Rule 4 and Rule $1+4$. Figure $3(\mathrm{~d})$ shows the different performances of Rule 4, Rule $1+4$, and random selection.
Experiment 5. Construct the training set using Rule_all. We compared the performance of Rule_all with Rules 2, 3, and 4 , as well as Rules $1+2,1+3$, and $1+4$, respectively. The results are shown in Figures 3(e) and 3(f).

It can be seen that we achieve a good performance by a limited-size training set. For example, when the size of training set is 150 (about $2.1 \%$ of the whole data), the classification error rate is about $18.2 \%$ under Rule_all criterion, while random selection only achieves an error rate around $33.8 \%$ with the same number of training samples.

To show the generalization ability of the proposed methods, we separate the entire dataset into two parts: the first part contains about 3500 shots, which are used for training set construction and training; the second part contains the remaining 3500 shots, which are used for testing. We construct the training set using all rules we proposed above, the comparisons of results are shown in Figure 4 . We can see when the size of training set is 300 (about $8.4 \%$ of the data used for training set construction), the classification error rate on the test dataset is about $18.8 \%$ under Rule_all criterion, while random selection only achieves an error rate around $34.3 \%$ with the same number of training samples.

All these experimental results demonstrate that these rules are effective for training set construction in video semantic classification and the hierarchical combination strategy could further improve the classification performance over each rule. However, this strategy could not improve the result of Rule $1+2$ significantly, which can be seen in Figures 3(f) and 4. The reasons for this phenomena lies in twofold: (1) the hierarchical strategy of combining the four rules in this paper is not the optimal solution, which still needs to be exploited in the future; (2) in this particular video collection, Rule $1+2$ removes most of the redundancy in the clustering information.

\section{CONCLUSIONS AND FUTURE WORK}

In this paper, we exploit the distribution characteristics of video dataset to construct efficient training set for video semantic classification. We proposed four metrics to reflect 
how well the constructed training set captures the distribution characteristics of the whole dataset; and the optimization rules for these metrics are further proposed based on these metrics. Experimental results demonstrate that these rules are effective, and obviously outperform random training set selection. For home video collections, maximizing temporal dispersiveness accompanied with salience is good enough since home videos tend to be temporally more similar than edited footages. However, for other datasets without such strong temporal similarity, such as the broadcast news videos, optimizing the other metrics that we proposed is still effective for training set construction.

Future work will be on the optimal combination of all these rules, as well as applying these rules on multiple semantic concepts, more types of videos, and larger video databases.

\section{ACKNOWLEDGMENT}

This work was performed when the first author was visiting Microsoft Research Asia as a research intern.

\section{REFERENCES}

[1] L. Xie, P. Xu, S.-F. Chang, A. Divakaran, and H. Sun, "Structure analysis of soccer video with domain knowledge and hidden markov models," Pattern Recognition Letters, vol. 25, no. 7, pp. 767-775, 2004.

[2] J. Fan, H. Luo, and X. Lin, "Semantic video classification by integrating flexible mixture model with adaptive em algorithm," in Proceedings of the ACM SIGMM International Workshop on Multimedia Information Retrieval, pp. 9-16, Berkeley, Calif, USA, November 2003.

[3] D. Zhong and S.-F. Chang, "Structure analysis of sports video using domain models," in Proceedings of IEEE International Conference in Multimedia \& Expo, pp. 713-716, Tokyo, Japan, August 2001.

[4] Y. Song, X.-S. Hua, L.-R. Dai, and M. Wang, "Semi-automatic video annotation based on active learning with multiple complementary predictors," in Proceedings of ACM SIGMM International Workshop on Multimedia Information Retrieval, pp. 97-104, Singapore, November 2005.

[5] R. Yan and M. Naphade, "Semi-supervised cross feature learning for semantic concept detection in videos," in Proceedings of the IEEE Computer Society Conference on Computer Vision and Pattern Recognition (CVPR '05), vol. I, pp. 657-663, 2005.

[6] J. Tang, X.-S. Hua, G.-J. Qi, M. Wang, T. Mei, and X. Wu, "Structure-sensitive manifold ranking for video concept detection," in Proceedings of ACM Multimedia, 2007.

[7] J. Tang, X.-S. Hua, T. Mei, G.-J. Qi, and X. Wu, "Video annotation based on temporally consistent gaussian random field," Electronics Letters, vol. 43, no. 8, pp. 448-449, 2007.

[8] M. Wang, Y. Song, X. Yuan, H.-J. Zhang, X.-S. Hua, and S. $\mathrm{Li}$, "Automatic video annotation by semi-supervised learning with kernel density estimation," in Proceedings of the 14th Annual ACM International Conference on Multimedia (MM '06), pp. 967-976, 2006.

[9] R. Yan, J. Yang, and A. Hauptmann, "Automatically labeling video data using multi-class active learning," in Proceedings of the IEEE International Conference on Computer Vision, vol. 1, pp. 516-523, Nice, France, October 2003.
[10] M.-Y. Chen, A. Hauptmann, M. Christel, and H. Wactlar, "Putting active learning into multimedia applications: dynamic definition and refinement of concept classifiers," in Proceedings of ACM International Conference on Multimedia, pp. 902-911, Singapore, November 2005.

[11] V. Vapnik, Three Remarks on Support Vector Method of Function Estimation. Advances in Kernel Methods: Support Vector Learning, MIT Press, 1999.

[12] J. Wu, X.-S. Hua, H.-J. Zhang, and B. Zhang, "An onlineoptimized incremental learning framework for video semantic classification," in Proceedings of the 12th ACM International Conference on Multimedia (ACM '04), pp. 320-323, New York, NY, USA, October 2004.

[13] K.-S. Goh, E. Chang, and W.-C. Lai, "Concept-dependent multimodal active learning for image retrieval," in Proceedings of the ACM International Conference on Multimedia, pp. 564571, New York, NY, USA, October 2004.

[14] G.-J. Qi, Y. Song, X.-S. Hua, L.-R. Dai, and H.-J. Zhang, "Video annotation by active learning and cluster tuning," in Proceedings of International Workshop on Semantic Learning Applications in Multimedia, vol. 2006, New York, NY, USA, June 2006.

[15] J. Yuan, J. Li, and B. Zhang, "Learning concepts from large scale imbalanced data sets using support cluster machines," in Proceedings of the 14th Annual ACM International Conference on Multimedia (MM '06), pp. 441-450, 2006.

[16] G. Baudat and F. Anouar, "Feature vector selection and projection using kernels," Neurocomputing, vol. 55, no. 1-2, pp. 21-38, 2003.

[17] “Trec video retrieval evaluation," http://www-nlpir.nist.gov/ projects/trecvid/.

[18] Y. Rui, T. S. Huang, M. Ortega, and S. Mehrotra, "Relevance feedback: a power tool for interactive content-based image retrieval," IEEE Transactions on Circuits and Systems for Video Technology, vol. 8, no. 5, pp. 644-655, 1998.

[19] C.-C. Chang and C.-J. Lin, "LIBSVM: a library for support vector machines," http://www.csie.ntu.edu.tw/ cjlin/libsvm/, 2001. 